\title{
MORPHOLOGICAL INSTABILITY OF SIMILARITY SOLUTION TO THE STEFAN PROBLEM WITH UNDERCOOLING AND SURFACE TENSION
}

\author{
BY \\ I. RUBINSTEIN AND B. ZALTZMAN \\ CEEP, Jacob Blaustein Institute for Desert Research, Ben-Gurion University of the Negev, \\ Sede-Boqer Campus, 84990, Israel
}

\begin{abstract}
This paper concerns morphological stability of a similarity solution to the Stefan problem with surface tension and initial supercooling. The linear stability analysis shows that for a nonzero surface tension each perturbation mode with a nonzero wave number is stable. However, the solution is unstable with respect to perturbations with a zero wave number limit point in their Fourier spectrum.
\end{abstract}

0. Introduction. In this paper we address the morphological stability of a similarity solution to the Stefan problem with surface tension and initial supercooling.

The study of shape instabilities in solidification with supercooling is important, in particular, because these instabilities initiate the appearance of dendrites.

Following the review by Langer [1], let us recall two well-known examples of morphological instabilities. The first concerns the quasi-steady-state growth of a spherical germ into a supercooled melt [2] (Mullins and Sekerka, 1963). The respective model problem reads:

$$
\begin{gathered}
u_{r r}+\frac{2}{r} u_{r}=0 \quad \forall r \neq R \\
\left.u\right|_{r=R}=-\frac{2 \gamma}{R}, \quad \gamma \text {-surface tension, } \\
\left.D\left[u_{r}\right]\right|_{r=R}=\frac{d R}{d t}
\end{gathered}
$$

(here [ ] denotes the jump across the solid/liquid interface (from solid to liquid)),

$$
u(\infty)=-\delta \text {. }
$$

(Hereon equal heat-diffusivities of the liquid and solid are assumed for the sake of simplicity.) There is a simple solution to this radial problem according to which a germ with an initial radius bigger than some critical value $R_{0}$ goes on growing monotonically. The

Received September 13, 1995.

1991 Mathematics Subject Classification. Primary 35B35, 35K05.

B. Zaltzman is a Sally Berg Foundation Fellow. 
linear stability analysis of this solution yields the existence of another critical value of the germ radius $R_{\mathrm{cr}}>R_{0}$, uniquely determined by $\gamma$ and $\delta$, above which the solution is unstable.

The next example we would like to recall concerns the travelling wave solution to the one-dimensional Stefan problem which, upon the transformation to a coordinate system moving with the wave speed $v$, reads

$$
\begin{gathered}
u_{z z}+v u_{z}=0 \quad \forall z \neq z_{0}, \\
\left.u\right|_{z=z_{0}}=0, \\
{\left[u_{z}\right]_{z_{0}+0}^{z_{0}-0}=v,} \\
u(\infty)=u_{\infty}, \quad u(-\infty)=0 .
\end{gathered}
$$

A one-parameter family of solutions (with arbitrary $v$ ) to this problem exists for only one critical value of undercooling: $u_{\infty}=-1$. For different undercoolings no planar travelling-wave solutions exist. The linear stability analysis of this solution yields a critical wave-length of perturbation, above which the solution is unstable. This critical wave-length depends on the wave speed, unspecified by undercooling. This sets the ground for the "selection" problem, resulting here from particularity of the basic solution concerned. In this sense, the aforementioned sphere growing into the undercooled melt represents a fairly exceptional example of an analytic solution to be subject to a stability analysis and completely determined by the data of the problem (undercooling).

Another explicit solution of the Stefan problem, specified completely by the undercooling, is the famous planar similarity solution of the two-phase problem. An additional importance of this solution lies in the fact that it represents the long-time asymptotics of the solutions to the one-dimensional Cauchy-Stefan problems with constant temperatures at infinities, whenever supercooling is below the critical value. In this solution the speed of the free boundary varies and tends to zero as time goes on. Stability of the planar similarity solution with respect to multidimensional perturbations of finite wave-length has been proved by L. Rubinstein [3] for the normal (non-supercooled) Stefan problem without surface tension. Chadam and Ortoleva [4] addressed stability of the respective solutions for the Stefan problem with surface tension and initial supercooling. They found that a similarity solution is unstable without surface tension and asymptotically stable when surface tension, however small, is present. More precisely, they proved that, with a nonvanishing surface tension, every perturbation mode with a finite nonzero wave number asymptotically decays in time.

We wish, however, to reexamine here the conclusion of the overall asymptotic stability of the planar similarity solution with supercooling in the presence of surface tension. Our ultimate claim is that a proper handling of the long-wave components of the perturbation results in instability for any given surface tension.

Notation. Hereon we will denote by $C$ with a respective subscript positive constants. We will also use the common notations $O(\cdot)$ and $o(\cdot)$, occasionally with subscripts denoting the respective infinitesimal variables. 
1. Formulation of the problem. Main results. The formulation of the problem is as follows. We consider a two-dimensional Stefan problem with surface tension:

$$
\begin{gathered}
u_{t}=\Delta u, \quad S(\mathbf{x}, t) \neq 0, \quad t>t_{0}, \\
u=-\gamma K, \quad[\nabla u \nabla S]=S_{t}, \quad \text { for } S(\mathbf{x}, t)=0, t>t_{0}, \\
u(\mathbf{x}, T)=u_{0}(\mathbf{x}), \quad S(\mathbf{x}, T)=S_{0}(\mathbf{x}), \\
u_{0}(\mathbf{x}) \rightarrow u_{\infty} \in(-1,0) \quad \text { as } x \rightarrow \infty, \quad u_{0}(\mathbf{x}) \rightarrow 0 \text { as } x \rightarrow-\infty .
\end{gathered}
$$

Here $S(\mathbf{x}, t)=0$ defines the position of the solid/liquid interface and $K(\mathbf{x}, t)$ its average curvature, $K=\left(|\nabla S|^{2} \Delta S-\frac{1}{2} \nabla\left(|\nabla S|^{2}\right) \cdot \nabla S\right) /\left(2|\nabla S|^{3}\right)$.

We are about to analyze the perturbation of the following planar similarity solution to the aforementioned problem:

$$
\begin{gathered}
S(\mathbf{x}, t)=\left\{x=R_{S}(t)=2 \alpha \sqrt{t}\right\}, \\
u^{S}= \begin{cases}u_{\infty}+2 \alpha \exp \left(\alpha^{2}\right)\left(1-\operatorname{erf}\left(\frac{x}{2 \sqrt{t}}\right)\right), & x>2 \alpha \sqrt{t} \\
0, & x<2 \alpha \sqrt{t} .\end{cases}
\end{gathered}
$$

Here $\alpha$ is the root of the transcendental equation

$$
2 \alpha \exp \left(\alpha^{2}\right) \int_{\alpha}^{\infty} \exp \left(-y^{2}\right) d y=-u_{\infty} .
$$

Let us consider a non-planar two-dimensional perturbation of the similarity solution of the form:

$$
\begin{gathered}
u_{\varepsilon}(x, y, t)=u^{S}(x, t)+\varepsilon u(x, y, t)+O\left(\varepsilon^{2}\right), \\
S(\mathbf{x}, t)=\left\{(x, y, t): x=R_{\varepsilon}(y, t)\right\}, \quad R_{\varepsilon}(y, t)=R_{S}(t)+\varepsilon R(y, t)+O\left(\varepsilon^{2}\right)
\end{gathered}
$$

with $R_{S}(t)=2 \alpha \sqrt{t}$.

The linearized initial-boundary value problem for the perturbation reads:

$$
\begin{gathered}
u_{t}=\Delta u, \quad x>R_{S}(t)=2 \alpha \sqrt{t}, \quad t>T \\
u=-\frac{\partial u^{S}}{\partial x} R+\frac{\gamma}{2} R_{y y}, \quad u_{x}=-R_{t}-\frac{\partial^{2} u^{S}}{\partial x^{2}} R, \quad \text { on } x=2 \alpha \sqrt{t}, t>T, \\
u(x, y, T)=\phi(x, y), \quad \text { for } x>2 \alpha \sqrt{T}, \quad \phi(x, y) \rightarrow 0 \text { as } x \rightarrow \infty, \quad R(y, T)=r(y) .
\end{gathered}
$$

Following Lev Rubinstein's idea [3] we make the change of the dependent variable

$$
v=u+\frac{\partial u^{S}}{\partial x} R-\frac{\gamma}{2} R_{y y}
$$

followed by the Fourier transform in $y$ :

$$
\begin{gathered}
w^{l} \stackrel{\text { def }}{=} \exp \left(l^{2} t\right) \int_{-\infty}^{\infty} v(x, y, t) \exp (-i l y) d y \\
f^{l}(t) \stackrel{\text { def }}{=} \int_{-\infty}^{\infty} R(y, t) \exp (-i l y) d y
\end{gathered}
$$


This yields the following one-dimensional time-dependent initial-boundary value problem for $w^{l}$ and $f^{l}$ :

$$
\begin{gathered}
w_{t}^{l}=w_{x x}^{l}+\left(u_{x}^{S}+\frac{\gamma^{l^{2}}}{2}\right)\left(\dot{f}^{l}+l^{2} f^{l}\right) \exp \left(l^{2} t\right), \quad x>2 \alpha \sqrt{t}, t>T, \\
w^{l}=0, \quad w_{x}^{l}=-\dot{f}^{l}(t) \exp \left(l^{2} t\right), \quad \text { for } x=2 \alpha \sqrt{t}, t>T, \\
w^{l}(x, T)=\phi^{l}(x), \quad f^{l}(T)=F(l) .
\end{gathered}
$$

Here $\phi^{l}(x)=\int_{-\infty}^{\infty}\left(\phi(x, y)+u_{x}^{S} r(y)-\frac{\gamma}{2} r_{y y}(y)\right) \exp (-i l y) d y, F(l)=\int_{-\infty}^{\infty} r(y) \exp (-i l y) d y$.

It may be shown (see Refs. [3], [4]), using the respective Green's function of the heat equation, that the solution satisfies the following integro-differential equation:

$$
\dot{f}^{l}(t)=-I(t) \exp \left(-l^{2} t\right)-\int_{T}^{t}\left(\dot{f}^{l}(t)+l^{2} f^{l}(t)\right) \exp \left(-l^{2}(t-\tau)\right)\left(P(t, \tau)+l^{2} \Gamma(t, \tau)\right) d \tau \text {. }
$$

Here $I(t)=U_{1 x}(2 \alpha \sqrt{t}, t)$ and $U_{1}$ is a solution of the following problem:

$$
\begin{gathered}
U_{1 t}=U_{1 x x}, \quad x>2 \alpha \sqrt{t}, t>T, \\
U_{1}(2 \alpha \sqrt{t}, t)=0, \quad t>T, \\
U_{1}(x, T)=\phi^{l}(x), \quad x>2 \alpha \sqrt{T} .
\end{gathered}
$$

Furthermore, $P(t, \tau)=U_{2 x}(2 \alpha \sqrt{t}, \tau)$ and $U_{2}$ is a solution of the problem

$$
\begin{gathered}
U_{2 t}=U_{2 x x}, \quad x>2 \alpha \sqrt{t}, t>\tau, \\
U_{2}(2 \alpha \sqrt{t}, t)=0, \quad t>\tau, \\
U_{2}(x, \tau)=u_{x}^{S}(x, \tau), \quad x>2 \alpha \sqrt{\tau} .
\end{gathered}
$$

$\Gamma(t, \tau)$ is defined by replacing $u_{x}^{S}(x, \tau)$ by $\frac{\gamma}{2}$ in the definition of $P(t, \tau)$.

Let us recall the following results of Refs. [3, 4], summarized below in Lemmas 1-3.

LEMMA 1. If $\phi^{l}(x)=O(1)$ as $x \rightarrow \infty$, then

$$
I(t) \leq C_{1} t^{-1 / 2} \text {. }
$$

Here the constant $C_{1}$ depends only on the bound on $\phi^{l}$ at infinity.

LEMMA 2. The following estimate holds:

$$
0<C_{2} \frac{\sqrt{\tau}}{t \sqrt{t-\tau}}<-P(t, \tau)<\frac{\alpha \sqrt{\tau}}{\sqrt{\pi} t \sqrt{t-\tau}} .
$$

For large $t, P(t, \tau)$ satisfies the asymptotic equality

$$
P(t, \tau)=-\frac{\alpha \sqrt{\tau}}{\sqrt{\pi} t \sqrt{t-\tau}}+\frac{\alpha^{2}}{2 t}+O(\sqrt{t-\tau})
$$

as $t-\tau \rightarrow 0+$.

LEMma 3. The following estimate holds:

$$
0<\frac{C_{3}}{\sqrt{t-\tau}}<\Gamma(t, \tau)<\frac{\gamma(1+\alpha)}{2 \sqrt{\pi} \sqrt{t-\tau}} .
$$


For large $t, \Gamma(t, \tau)$ satisfies the asymptotic equality

$$
\Gamma(t, \tau)=\frac{\gamma}{2 \sqrt{\pi} \sqrt{t-\tau}}+\frac{\alpha \gamma}{4 \sqrt{t}}+O(\sqrt{t-\tau})
$$

as $t-\tau \rightarrow 0+$.

The following Theorem 1 is a straightforward modification of the main Theorem 4.1 of Ref. [4], proved there by using the asymptotic properties (1.24), (1.26), (1.28) of the kernels $P(t, \tau), \Gamma(t, \tau)$ and the function $I(t)$.

THEOREM 1. For each fixed $l>0$, the following equality holds:

$$
f^{l}(t)=C_{4}(l) g^{l}(t) \exp \left(-b^{2} t\right)(1+o(1)), \quad \text { as } l^{2} t \rightarrow \infty .
$$

Here

$$
g^{l}(t) \stackrel{\text { def }}{=} t^{\alpha \sqrt{l^{2}-b^{2}} /\left(4\left(1+b^{2}\right)\right)} \exp \left(\frac{\alpha \sqrt{l^{2}-b^{2}}}{2\left(1+b^{2}\right)} \sqrt{t}\right)
$$

is the solution of the following asymptotic equation:

$$
\dot{g}^{l}\left(1+b^{2}-\frac{\alpha}{2 \sqrt{l^{2}-b^{2}} \sqrt{t}}\right)=\frac{\alpha \sqrt{l^{2}-b^{2}}}{2 \sqrt{t}} g^{l}
$$

and

$$
b^{2} \stackrel{\text { def }}{=}-\frac{\gamma^{2} l^{4}}{8}+\frac{l^{3} \gamma \sqrt{16+\gamma^{2} l^{2}}}{8} .
$$

Hereon, for the sake of simplicity, we limit ourselves to odd perturbations and thus to a sine Fourier transform with $l$ nonnegative. The limitation may be trivially lifted by replacing in what follows $l$ by $|l|$.

REMARK. For large $t$ and small $l$ the following approximate equality holds:

$$
\begin{gathered}
g^{l}=\exp (\alpha l \sqrt{t})\left(l^{2} t\right)^{\alpha^{2}}(1+o(1)) \quad \text { as } l \rightarrow 0, \\
f^{l}=C_{4} \exp (\alpha l \sqrt{t}) \exp \left(-\gamma l^{3} t\right)\left(l^{2} t\right)^{\alpha^{2}}\left(1+o_{l}(1)+o_{l^{2} t}(1)\right) \quad \text { as } l \rightarrow 0, l^{2} t \rightarrow \infty
\end{gathered}
$$

Using these equalities Chadam and Ortoleva [4] concluded that for every $\gamma>0$ the similarity solution is stable, since $f \rightarrow 0$ when $t \rightarrow \infty$ for every $l>0$. One may, however, distinguish two time scales as defined by this equality: for $t$ such that $l^{3} t \ll 1, f^{l}$ grows exponentially in $\sqrt{t}$, whereas for $t$ such that $l^{4} t \gg 1$ it decays exponentially. Thus, for $l$ sufficiently small the stabilization time is very large.

This motivates the following hypothesis: the neighborhood of zero of the perturbation Fourier spectrum may have an effect upon the solution stability. To analyze this effect, we have to study not a fixed mode of perturbation, but rather investigate all modes of perturbation, especially the long-wave part of the Fourier spectrum, uniformly in time.

For this purpose one should look for a suitable scaling of the time and space variables. Let us define the scaled variables as

$$
\tau=t l^{2}, \quad z=x l, \quad W^{l}=\frac{w^{l}}{l} .
$$


The function $W^{l}$ satisfies the following initial-boundary value problem:

$$
\begin{gathered}
W_{\tau}^{l}=W_{z z}^{l}+u_{z}^{S}\left(\dot{f}^{l}+f^{l}\right) \exp (\tau)+\frac{\gamma l}{2}\left(\dot{f}^{l}+f^{l}\right) \exp (\tau), \quad z>2 \alpha \sqrt{\tau}, \tau>l^{2} T \\
W^{l}=0, \quad W_{z}^{l}=-\dot{f}^{l}(\tau) \exp (\tau), \quad \text { for } z=2 \alpha \sqrt{\tau}, \tau>l^{2} T \\
W^{l}\left(z, l^{2} T\right)=\frac{\phi^{l}(z)}{l}, \quad f^{l}\left(l^{2} T\right)=F(l) .
\end{gathered}
$$

$l$ enters crucially this scaled formulation through:

1. the initial moment dependence $l^{2} T$,

2. the inhomogeneous term $\frac{\gamma^{l}}{2}\left(\dot{f}^{l}+f^{l}\right) \exp (\tau)$ in the equation (1.35).

The function $f^{l}$ satisfies the following integro-differential equation with respect to the new time variable $\tau$ :

$$
\dot{f}^{l}(\tau)=-\widetilde{I}(\tau) \exp (-\tau)-\int_{l^{2} T}^{\tau}\left(\dot{f}^{l}(\tau)+f^{l}(\tau)\right) \exp (-(t-\tau))(P(\tau, s)+l \Gamma(\tau, s)) d s .
$$

Here $\widetilde{I}(\tau)$ is defined by replacing $\phi^{l}(x)$ by $\frac{\phi^{l}(z)}{l}$ in the definition of $I(t)$.

The possibility to approximate, for $\tau \ll \frac{1}{l}$, the solution of the problem $(1.35)-(1.37 \mathrm{a}, \mathrm{b})$ by that of the limiting problem for $l \rightarrow 0$ is asserted by the following Theorem.

Theorem 2. If $\phi^{l}(z)=O\left(l^{2}\right)$ and $\phi_{z}^{l}(z)=O(l)$ uniformly in $z$ as $l \rightarrow 0$ then for $\forall \tau \in\left[0, \frac{1}{l^{1-\varepsilon}}\right], \varepsilon>0$,

$$
f^{l}=F(l) f_{0}(\tau)\left(1=O\left(l^{\varepsilon}\right)\right) \quad \text { as } l \rightarrow 0 .
$$

Here $f_{0}$ and $W_{0}$ are the solutions of the following limiting initial-boundary problem independent of $l$ :

$$
\begin{gathered}
W_{0 \tau}=W_{0 z z}+u_{z}^{S}\left(\dot{f}^{0}+f^{0}\right) \exp (\tau), \quad z>2 \alpha \sqrt{\tau}, \tau>0, \\
W_{0}=0, \quad W_{0 z}=-\dot{f}_{0}(\tau) \exp (\tau), \quad z=2 \alpha \sqrt{\tau}, \tau>0, \\
W_{0}(z, 0)=0, \quad f_{0}(0)=1 .
\end{gathered}
$$

Proof of Theorem 2. The proof is based on the repeated use of the monotonicity property of the integro-differential operator (1.38) expressed by the following Lemma:

LEMMA 4. a) $-P(t, \tau)>l \Gamma(t, \tau)$ for $\forall \tau \in\left[T_{0}, t\right], \forall t \in\left[0, C_{5} \frac{\sqrt{T_{0}}}{l}\right]$.

b) Let $I(t) \leq 0, \dot{f}\left(T_{0}\right)>0, f\left(T_{0}\right)>0$. Then $f(t), \dot{f}(t)>0 \forall t \in\left[T_{0}, \frac{C_{5} \sqrt{T_{0}}}{l}\right]$.

Proof of Lemma 4. Since

$$
U_{2}(x, t, \tau)=\frac{1}{\sqrt{\tau}} U_{2}\left(\frac{x}{\sqrt{\tau}}, \frac{t}{\tau}, 1\right), \quad U_{2 x}(x, t, \tau)=\frac{1}{\tau} U_{2 x}\left(\frac{x}{\sqrt{\tau}}, \frac{t}{\tau}, 1\right),
$$

then

$$
P(t, \tau)=\frac{1}{\tau} U_{2 x}\left(2 \alpha \sqrt{\frac{t}{\tau}}, \frac{t}{\tau}, 1\right)=\frac{1}{\tau} \widetilde{P}\left(\frac{t}{\tau}\right)
$$


Applying the same change of variables to the function $\Gamma(t, \tau)$, we find that

$$
\Gamma(t, \tau)=\frac{1}{\sqrt{\tau}} U_{3 x}\left(2 \alpha \sqrt{\frac{t}{\tau}}, \frac{t}{\tau}, 1\right)=\frac{1}{\sqrt{\tau}} \widetilde{\Gamma}\left(\frac{t}{\tau}\right) .
$$

Therefore, we have to compare the functions $\frac{1}{\sqrt{\tau}} \widetilde{P}(\xi)$ and $l \widetilde{\Gamma}(\xi)$ for $\xi>1$. Making use of the estimates (1.25), (1.27), we obtain that the inequality

$$
-\frac{1}{\sqrt{t}} \widetilde{P}(\xi)>l \widetilde{\Gamma}(\xi)>0
$$

holds for all $\xi>1$ such that

$$
l \sqrt{t} \xi<\frac{2 \sqrt{\pi}}{\gamma(1+\alpha)} C_{2} .
$$

Returning to the variables $t$ and $\tau$, we obtain

$$
0<t<\frac{2 \sqrt{\pi}}{\gamma(1+\alpha)} C_{2} \frac{\sqrt{T_{0}}}{l} .
$$

Choosing $C_{5}=\frac{2 \sqrt{\pi}}{\gamma(1+\alpha)} C_{2}$ we complete the proof of the statement a) of Lemma 4 .

Let us prove the statement b). Since $\dot{f}\left(T_{0}\right)>0, t^{*}=\inf _{t>T_{0}}(\dot{f}(t) \leq 0)>T_{0}$. Making use of the statement a) we obtain the inequality $t^{*}>C_{5} \sqrt{T_{0}} / l$, thus completing the proof of Lemma 4.

The proof of Theorem 2 proceeds by considering three overlapping time intervals: short time $\left(l^{2} T \leq \tau \leq T^{*}\right)$, intermediate time $\left(\frac{T^{*}}{2} \leq \tau \leq 4 \alpha^{2}\right)$, and long time $\left(2 \alpha^{2} \leq \tau \ll \frac{1}{l}\right)$. The appearance of these time intervals is motivated by the presence of two singular points in the equation (1.35), $\tau=0$ and $\tau=\infty$. We will prove Theorem 2 by constructing the respective barrier functions in, generally speaking, nonoverlapping neighborhoods of these singular points. Therefore, in order to complete the estimate (1.39) for all times we will also have to consider the intermediate time.

LEMma 5 (Short time estimates $l^{2} T \leq \tau \leq T^{*}$ ). If the conditions of Theorem 2 hold, then for $\forall \tau \in\left[l^{2} T, T^{*}\right]$

$$
W^{l}=F(l) W_{0}(1+O(l)), \quad f^{l}=F(l) f_{0}(\tau)(1+O(l)) \quad \text { as } l \rightarrow 0,
$$

where $T^{*}$ is a positive constant independent of $l$.

Proof of Lemma 5. Returning to Lemma 4 we observe that the integro-differential operator (1.38) is monotonic for $\tau \in\left[l^{2} T, C_{5} \sqrt{T}\right]$. Thus we base the proof of Lemma 5 upon the construction of the respective barrier functions. Let us define the lower barrier function $V$ as a solution of the following boundary value problem:

$$
\begin{gathered}
V_{\tau}=V_{z z}+\frac{u_{z}^{S} \dot{H}}{\beta}, \quad z>2 \alpha \sqrt{\tau}, \tau>0, \\
V=0, \quad V_{z}=-\beta \dot{H}, \quad \text { for } z=2 \alpha \sqrt{\tau}, \tau>0 .
\end{gathered}
$$

Here $0<\beta<1$ is a constant defined below. We define the upper barrier function as $-V$. 
We look for $V$ in the family of self-similar solutions:

$$
V=V(\xi)=V\left(\frac{z}{2 \sqrt{\tau}}\right), \quad H=C_{6} \sqrt{\tau} .
$$

Since $u_{z}^{S}=\alpha \exp \left(\alpha^{2}-\xi^{2}\right) / \sqrt{\tau}$, the function $V$ satisfies the following differential equation:

$$
V^{\prime \prime}+2 \xi V^{\prime}=\frac{2 \alpha}{\beta} C_{6} \exp \left(\alpha^{2}-\xi^{2}\right), \quad \xi>\alpha .
$$

Integration of this equation yields

$$
V^{\prime}=C_{6} \frac{2 \alpha}{\beta}(\xi-\alpha) \exp \left(\alpha^{2}-\xi^{2}\right)-C_{7} \exp \left(-\xi^{2}\right)
$$

Through another integration, making use of the boundary conditions, we obtain

$$
V=C_{6} \frac{\alpha}{\beta} \exp \left(\alpha^{2}\right)\left(-\sqrt{\pi}\left(\frac{\beta^{2}}{2 \alpha}+\alpha\right)(\operatorname{erf}(\xi)-\operatorname{erf}(\alpha))+\exp \left(-\alpha^{2}\right)-\exp \left(-\xi^{2}\right)\right)
$$

Let us prove that for $\forall \alpha>0$ there exists $\beta \in(0,1)$ such that $V(\xi)<0$ for $\forall \xi>\alpha$. Since $V(\alpha)=0, V^{\prime}(\xi)<0$ for $\xi \in\left(\alpha, \alpha+\frac{\beta^{2}}{2 \alpha}\right), V^{\prime}(\xi)>0$ for $\xi>\alpha+\frac{\beta^{2}}{2 \alpha}$ it is enough to prove the aforementioned statement for $\xi=+\infty$ :

$$
V(+\infty)=C_{6} \frac{\alpha}{\beta} \exp \left(\alpha^{2}\right)\left(\exp \left(-\alpha^{2}\right)-\sqrt{\pi}\left(\frac{\beta^{2}}{2 \alpha}+\alpha\right)(1-\operatorname{erf}(\alpha))\right)
$$

Since

$$
\frac{\sqrt{\pi}}{2} \exp \left(\alpha^{2}\right)(1-\operatorname{erf}(\alpha))>\frac{1}{\sqrt{\alpha^{2}+2}+\alpha}
$$

(see Ref. [5], p. 298),

$$
V(+\infty)<-C_{6} \frac{\alpha}{\beta} \frac{\frac{\beta^{2}}{\alpha}-\sqrt{\alpha^{2}+2}}{\sqrt{\alpha^{2}+2}+\alpha} .
$$

The latter estimate yields

$$
V(+\infty)<0 \quad \text { for } \forall \beta \in\left(\frac{2 \alpha}{\sqrt{\alpha^{2}+2}+2}, 1\right) .
$$

In order to show that $V(z, \tau)$ and $-V(z, \tau)$ indeed represent the lower and upper barriers, respectively, for $W^{l}(z, \tau)$ we use the following construction. Let us begin with evaluating $W_{0}, f_{0}$ at the initial moment $\tau=l^{2} T_{0}$. To this end, let us define the functions $\widetilde{W}(z, \tau) \stackrel{\text { def }}{=}-W_{0}(z, \tau)+l V(z, \tau)$ and $\widetilde{H} \stackrel{\text { def }}{=}-\left(f_{0}(\tau)-1\right)+l H(\tau)$, satisfying the equation

$$
\widetilde{W}_{\tau}=\widetilde{W}_{z z}+u_{z}^{S}(\dot{\widetilde{H}}+\widetilde{H}) \exp (\tau)+J_{1}(z, \tau)
$$

where

$$
J_{1}=u_{z}^{S}\left(l \dot{H}\left(\frac{1}{\beta}-\exp (\tau)\right)-l H \exp (\tau)-\exp (\tau)\right)
$$


For the function $l V$ to be lower barrier for $W_{0}$ on the time interval $\left(0, l^{2} T\right), \widetilde{W}$ has to be negative and for this it suffices for $J_{1}$ to be negative for $\tau \in\left(0, l^{2} T\right)$. The latter is guaranteed, taking into account that $u_{z}^{S}$ is negative, by the inequality

$$
J_{1}<u_{z}^{S}\left(C_{6} \frac{1}{2 \sqrt{T}}\left(\frac{1}{\beta}-\exp \left(l^{2} T\right)\right)-l^{2} \sqrt{T} \exp (\tau)-\exp \left(l^{2} T\right)\right)<0 \quad \text { for } \tau \in\left(0, l^{2} T\right) .
$$

Obviously, (1.62) holds for $C_{6}>0$ sufficiently large. Making use of boundary conditions (1.41b) (1.51b) and inequality (1.62) we obtain

$$
\dot{\tilde{H}}(\tau)>-l I_{1}(\tau) \exp (-\tau)-\int_{0}^{\tau}(\dot{\widetilde{H}}(s)+\widetilde{H}(s)) \exp (-(s-\tau)) P(\tau, s) d s,
$$

where $I_{1}(\tau)$ is defined by replacing $\phi^{l}(x)$ by $V_{\infty}=\lim _{z \rightarrow \infty} V(z, \tau)<0$ in the definition of $I(t)$. Similarly to the proof of statement b) of Lemma 4 , we find that $\dot{\widetilde{H}}>0$, which implies through integration, using the definition of $\widetilde{H}$,

$$
f_{0}(\tau)-1<C_{6} l^{2} \sqrt{T} \text { for } \forall \tau \in\left[0, l^{2} T\right] .
$$

Applying the maximum principle to Eq. (1.60) and accounting for the Dirichlet boundary data and estimates (1.62), (1.64), we obtain

$$
\widetilde{W}(z, \tau)<0 \Rightarrow W_{0}(z, \tau) \geq V_{\infty} l \quad \text { for } \forall z>2 \alpha \sqrt{\tau}, \tau \in\left[0, l^{2} T\right] .
$$

Similarly, we may consider the sums $W_{0}(z, \tau)+l V(z, \tau)$ and $\left(f_{0}(\tau)-1\right)+l H(\tau)$ and obtain the following estimate:

$$
\left|f_{0}(\tau)-1\right|<C_{6} l^{2} \sqrt{T}, \quad\left|W_{0}(z, \tau)\right| \leq-V_{\infty} l \quad \text { for } \forall z>2 \alpha \sqrt{\tau}, \tau \in\left[0, l^{2} T\right] .
$$

The estimates $(1.66 \mathrm{a}, \mathrm{b})$ allow us to consider the differences $W^{l}-F(l) W_{0} \stackrel{\text { def }}{=} W_{1}(z, \tau)$, $f^{l}-F(l) f_{0} \stackrel{\text { def }}{=} f_{1}(\tau)$ for $\tau>l^{2} T$. The conditions of Lemma 5 and estimates $(1.66 \mathrm{a}, \mathrm{b})$ yield

$$
\left|W_{1}\left(z, l^{2} T\right)\right|<C_{8} l V\left(z, l^{2} T\right) \quad \text { for } z \geq l^{2} T, \quad\left|f_{1}\left(l^{2} T\right)\right|<C_{8} l H\left(l^{2} T\right)
$$

for some $C_{8}>0$. Let us consider the differences $C_{8} l V(z, \tau)-W_{1}(z, \tau) \stackrel{\text { def }}{=} W_{2}(z, \tau)$, $C_{8} l H(\tau)-f_{1}(\tau) \stackrel{\text { def }}{=} f_{2}(\tau)$. These functions satisfy the following equations:

$$
W_{2 \tau}=W_{2 z z}+u_{z}^{S}\left(\dot{f}_{2}+f_{2}\right) \exp (\tau)+\frac{\gamma l}{2}\left(\dot{f}_{2}+f_{2}\right) \exp (\tau)+J_{2}(z, \tau)
$$

where

$$
\begin{aligned}
J_{2}(z, \tau)= & C_{8}\left(u_{z}^{S}\left(\dot{H}\left(\frac{1}{\beta}-\exp (\tau)\right)-H \exp (\tau)\right)-\frac{\gamma l}{2}(\dot{H}+H) \exp (\tau)\right) \\
& +\frac{\gamma l}{2}\left(\dot{f}_{0}+f_{0}\right) \exp (\tau) .
\end{aligned}
$$

Once again, it is observed by comparing (1.35) and (1.68) that for the function $C_{8} l V$ to be a lower barrier for $W_{1}$, it suffices for $J_{2}$ to be negative. Since it may be easily shown, similarly to (1.64) that for $C_{8}$ sufficiently large

$$
C_{8}(\dot{H}+H)-\left(\dot{f}_{0}+f_{0}\right)>0 \text { for } \tau \in\left(l^{2} T, \frac{C_{5}}{\sqrt{T}}\right),
$$


the negativity of $J_{2}$ is guaranteed, taking into account that $u_{z}^{S}$ is negative, by the inequality

$$
\dot{H}\left(\frac{1}{\beta}-\exp (\tau)\right)-H \exp (\tau)>0 .
$$

Obviously, (1.71) holds for $0<\tau<C_{9}$ for some $C_{9} \in\left(0, C_{5} / \sqrt{T}\right)$. Hereon, the rest of the proof of Lemma 5 is identical to the proof of estimates $(1.66 \mathrm{a}, \mathrm{b})$; that is, omitting the unneccessary technicalities, the respective integro-differential inequality for the function $f_{2}$ is written and the monotonicity properties of Lemma 4 are used; upon obtaining the estimate $(1.49 \mathrm{~b})$, we use the maximum principle to estimate the function $W_{2}$.

LEMMA 6 (Intermediate time estimates $T^{*} / 2 \leq \tau \leq 4 \alpha^{2}$ ). If the conditions of Theorem 2 hold, then for $\forall \tau \in\left[\frac{T^{*}}{2}, 4 \alpha^{2}\right]$

$$
W^{l}=F(l) W_{0}(1+O(l)), \quad f^{l}=F(l) f_{0}(\tau)(1+O(l)) \quad \text { as } l \rightarrow 0 .
$$

Proof of Lemma 6. With the help of Lemma 5, we can neglect the dependence of the initial moment on $l$ and consider the problem (1.34)-(1.36) with the initial moment $T^{*} / 2$ and the following estimates on the initial data:

$$
\begin{array}{r}
W^{l}\left(z, \frac{T^{*}}{2}\right)=F(l) W_{0}\left(z, \frac{T^{*}}{2}\right)(1+O(l)), \quad f^{l}\left(\frac{T^{*}}{2}\right)=F(l) f_{0}\left(\frac{T^{*}}{2}\right)(1+O(l)) \\
\text { as } l \rightarrow 0 .
\end{array}
$$

Lemma 4 yields the monotonicity of operator (1.38) in the time interval $\left[T^{*} / 2,4 \alpha^{2}\right]$. Let us consider the functions

$$
Q(z, \tau)=\frac{W^{l}-F(l) W_{0}}{l}, \quad E(\tau)=\frac{f^{l}-F(l) f_{0}(\tau)}{l} .
$$

According to (1.35)-(1.36) and (1.40)-(1.41), they are solutions of the following initialboundary value problem:

$$
\begin{gathered}
Q_{\tau}=Q_{z z}+u_{z}^{S}(\dot{E}+E) \exp (\tau)+\frac{\gamma^{l}}{2}(\dot{E}+E)+\frac{\gamma}{2} F(l)\left(\dot{f}_{0}(\tau)+f_{0}(\tau)\right), \\
\tau>T^{*}, z>2 \alpha \sqrt{\tau}, \\
Q=0, \quad Q_{z}=-\dot{E} \exp (\tau), \quad \tau>T^{*}, \quad z=2 \alpha \sqrt{\tau} .
\end{gathered}
$$

Since Lemma 5 yields the initial boundedness of the functions $Q$ and $E$ at the instant $T^{*} / 2$, one may easily prove, using the respective barrier functions, boundedness of the functions $Q$ and $E$ in the finite time interval $\tau \in\left[\frac{T^{*}}{2}, 4 \alpha^{2}\right]$. The upper barrier may be defined as the solution of the equation (1.75) with $l$ equal to zero and positive initial data. The proof of Lemma 6 is thus completed.

LEMMA 7 (Long time estimates $4 \alpha^{2} \leq \tau \ll 1 / l$ ). If the conditions of Theorem 2 hold, then for $\forall \tau \in\left[2 \alpha^{2}, 1 / l^{1-\varepsilon}\right], \forall \varepsilon \in(0,1)$,

$$
f^{l}=F(l) f_{0}(\tau)\left(1+O\left(l^{\varepsilon}\right)\right) \quad \text { as } l \rightarrow 0 .
$$


Proof of Lemma 7. With the help of Lemma 6 we can consider the problem (1.35)(1.37) with the initial moment equal to $2 \alpha^{2}$. Lemma 4 yields the monotonicity of operator (1.17) in the time interval $\left[2 \alpha^{2}, 1 / l^{1-\varepsilon}\right]$.

Let us once more consider the functions $Q(z, \tau), E(\tau)$ defined in the proof of Lemma 6. The functions $E(\tau)$ and $f_{0}(\tau)$ satisfy the following integro-differential equations:

$$
\begin{aligned}
\dot{f}_{0}(\tau)=-I_{1}(\tau) \exp (-\tau)-\int_{2 \alpha^{2}}^{\tau}\left(\left(\dot{f}_{0}(s)+f_{0}(s)\right) \exp (-(\tau-s)) P(\tau, s) d s\right. \\
\dot{E}(\tau)=-I_{2}(\tau) \exp (-\tau)-\int_{2 \alpha^{2}}^{\tau}((\dot{E}(s)+E(s)) \exp (-(\tau-s))(P(\tau, s)+l \Gamma(\tau, s)) \\
\left.+F(l)\left(\dot{f}_{0}(s)+f_{0}(s)\right) \Gamma(\tau, s)\right) d s
\end{aligned}
$$

Here $I_{1}(\tau)$ and $I_{2}(\tau)$ are defined by replacing $\phi^{l}(x)$ by $W_{0}\left(z, 2 \alpha^{2}\right)$ and $\left(W^{l}\left(z, 2 \alpha^{2}\right)-\right.$ $\left.F(l) W_{0}\left(z, 2 \alpha^{2}\right)\right) / l$, respectively, in the definition of $I(t)$. Making use of Lemmas 1 and 6 we obtain the estimate

$$
\left|I_{1}(\tau)\right|<\frac{C_{10}}{\sqrt{\tau}}, \quad\left|I_{2}(\tau)\right|<\frac{C_{10}}{\sqrt{\tau}}
$$

Let us define the functions $f_{1}, f_{2}$ as solutions of the following asymptotic equations:

$$
\begin{gathered}
\dot{f}_{1}=\frac{C_{10}}{\sqrt{\tau}} \exp (-\tau)+\frac{\alpha}{\sqrt{\tau}}\left(\dot{f}_{1}+f_{1}\right), \quad f_{1}\left(2 \alpha^{2}\right)=2 f_{0}\left(2 \alpha^{2}\right) \\
\dot{f}_{2}=\frac{C_{10}}{\sqrt{\tau}} \exp (-\tau)+\frac{\alpha}{\sqrt{\tau}}\left(\dot{f}_{2}+f_{2}\right)+C_{11}(\gamma(1+\alpha))\left(\dot{f}_{1}+f_{1}\right), \quad f_{2}\left(2 \alpha^{2}\right)=2\left|E\left(2 \alpha^{2}\right)\right|
\end{gathered}
$$

where $C_{11}=\max _{l} F(l)$.

Equations (1.81), (1.82) and estimates $(1.80 \mathrm{a}, \mathrm{b})$ yield

$$
\begin{gathered}
\dot{f}_{1}(\tau) \geq\left|I_{1}(\tau)\right| \exp (-\tau)+\left(\dot{f}_{1}(\tau)+f_{1}(\tau)\right) \int_{2 \alpha^{2}}^{\infty} \frac{\alpha \sqrt{s}}{k \sqrt{\pi} \tau \sqrt{\tau-s}} \exp (-(\tau-s)) d s \\
\dot{f}_{2}(\tau) \geq\left|I_{2}(\tau)\right| \exp (-\tau)+\left(\dot{f}_{2}(\tau)+f_{2}(\tau)\right) \int_{2 \alpha^{2}}^{\infty} \frac{\alpha \sqrt{s}}{k \sqrt{\pi} \tau \sqrt{\tau-s}} \exp (-(\tau-s)) d s \\
+C_{11}\left(\dot{f}_{1}(\tau)+f_{1}(\tau)\right) \int_{2 \alpha^{2}}^{\infty} \frac{\gamma(1+\alpha)}{2 \sqrt{\pi} \sqrt{\tau-s}} \exp (-(\tau-s)) d s
\end{gathered}
$$

It may be shown by differentiation of (1.81), (1.82) that for $\tau$ large enough, $\ddot{f}_{1}, \ddot{f}_{2}>0$. Using the monotonic growth of the functions $\dot{f}_{2}, f_{2}, \dot{f}_{1}, f_{1}$ in time and the inequalities (1.25), (1.27) we obtain the following integral inequalities:

$$
\begin{gathered}
f_{1}(\tau) \geq\left|I_{1}(\tau)\right| \exp (-\tau)-\int_{2 \alpha^{2}}^{\tau}\left(\dot{f}_{1}(s)+f_{1}(s)\right) \exp (-(\tau-s)) P(\tau, s) d s \\
\dot{f}_{2}(\tau) \geq\left|I_{2}(\tau)\right| \exp (-\tau)-\int_{2 \alpha^{2}}^{\tau}\left(\left(\dot{f}_{2}(s)+f_{2}(s)\right) P(\tau, s)+l\left(\dot{f}_{2}(s)+f_{2}(s)\right) \Gamma(\tau, s)\right. \\
\left.-C_{11}\left(\dot{f}_{1}(s)+f_{1}(s)\right) \Gamma(\tau, s)\right) \exp (-(\tau-s)) d s
\end{gathered}
$$


Comparing (1.78) and (1.85) we obtain the following inequality for the difference $f_{1}-f_{0}$ :

$$
\dot{f}_{1}(\tau)-\dot{f}_{0}(\tau) \geq-\int_{2 \alpha^{2}}^{\tau}\left(\left(\dot{f}_{1}(s)-\dot{f}_{0}(s)\right)+\left(f_{1}(s)-f_{0}(s)\right)\right) \exp (-(\tau-s)) P(\tau, s) d s .
$$

Making use of the statement b) of Lemma 4 and the positivity of the difference $f_{1}-f_{0}$ at the instant $\tau=2 \alpha^{2}$ we conclude that

$$
\dot{f}_{0} \leq \dot{f}_{1}, \quad f_{0} \leq f_{1} \quad \text { for } \tau \geq 2 \alpha^{2} .
$$

Accounting for the latter inequalities, we may rewrite the inequality (1.86) in the following form:

$$
\begin{aligned}
\dot{f}_{2}(\tau) \geq & \left|I_{2}(\tau)\right| \exp (-\tau)-\int_{2 \alpha^{2}}^{\tau}\left(\left(\dot{f}_{2}(s)+f_{2}(s)\right) P(\tau, s)+l\left(\dot{f}_{2}(s)+f_{2}(s)\right) \Gamma(\tau, s)\right. \\
& \left.-\left(\dot{f}_{0}(s)+f_{0}(s)\right) \Gamma(\tau, s)\right) \exp (-(\tau-s)) d s .
\end{aligned}
$$

Let us denote by $G(\tau)$ the difference $f_{2}(\tau)-E(\tau)$. Using the inequality (1.89) we obtain

$$
\begin{aligned}
\dot{G}(\tau) \geq & -\int_{2 \alpha^{2}}^{\tau}((\dot{G}(s)+G(s)) P(\tau, s)+l(\dot{G}(s)+G(s)) \Gamma(\tau, s) \\
& -l(\dot{G}(s)+G(s)) \Gamma(\tau, s)) \exp (-(\tau-s)) d s .
\end{aligned}
$$

Once more, making use of the statement b) of Lemma 4 and the positivity of $G$ at the instant $\tau=2 \alpha^{2}$, we conclude that

$$
E(\tau)<f_{2}(\tau) \text { for } \tau \in\left[2 \alpha^{2}, \frac{1}{l^{1-\varepsilon}}\right] .
$$

Similarly, we may consider the sum $f_{2}(\tau)+E(\tau)$ and obtain the following estimate:

$$
|E(\tau)|<f_{2}(\tau) \text { for } \tau \in\left[2 \alpha^{2}, \frac{1}{l^{1-\varepsilon}}\right] \text {. }
$$

Since

$$
0<f_{2}(\tau) \leq C_{12} \tau f_{1}(\tau)
$$

whereas Theorem 1 yields

$$
f_{0}(\tau)=f_{1}(\tau)\left(C_{13}+o(1)\right) \text { as } \tau \rightarrow 0,
$$

we conclude that

$$
\left|f^{l}(\tau)-f_{0}(\tau)\right|=l|E(\tau)| \leq \frac{C_{14} l \tau}{1+o(1)} f_{0}(\tau) \quad \text { as } \tau \rightarrow 0 .
$$

This completes the proof of Lemma 7.

By this we have also completed the proof of Theorem 2 .

Making use of the equality (1.29), asymptotically valid for $\tau \gg 1$, and returning to the original variables we obtain the following main theorem.

Theorem 3. Let $F(l)=\int_{-\infty}^{\infty} R(y, T) \exp (-i l y) d y$ and $F(0)=0, F(\infty)=0, F^{(n)}(0) \neq$ 0 for some $n$. Then if $\phi^{l}(x)=O\left(l^{2}\right), \phi_{x}^{l}(x)=O\left(l^{2}\right)$ as $l \rightarrow 0$ uniformly in $x$, the perturbation of the free boundary grows unboundedly in time. 
Proof of Theorem 3. The idea of the proof is as follows. The overlap of the two time intervals $\tau \ll \frac{1}{l}$ and $\tau \gg 1$, which appeared in Theorems 1 and 2, yields the Fourier transform of the free boundary $f^{l}(t)=\int_{-\infty}^{\infty} R(y, t) \exp (-i l y) d y$ in the form

$$
f^{l}(t)=C_{15} F(l) \exp (\alpha l \sqrt{t}) \exp \left(-\frac{\gamma l^{3} t}{2}\right)\left(l^{2} t\right)^{\alpha^{2}}\left(1+o_{l^{2} t}(1)+o_{l}(1)\right)
$$

as $\tau=l^{2} t \rightarrow \infty, l \rightarrow 0$. Making use of the inverse Fourier transform we shall then prove the statement of Theorem 3 .

Equality (1.95) is obtained by using Theorems 1 and 2 which yield

$$
f^{l}(\tau)=C_{15} F(l) g(\tau)\left(1+o_{\tau}(1)\right)\left(1+o_{l}(1)\right) \quad \text { as } \tau \rightarrow \infty, l \rightarrow 0 .
$$

Furthermore, Remark 1 to Theorem 1 yields

$$
f^{l}(\tau)=C_{15} F(l) \exp (\alpha \sqrt{\tau}) \exp \left(-\frac{\gamma l t}{2}\right)(\tau)^{\alpha^{2}}\left(1+o_{\tau}(1)+o_{l}(1)\right) \quad \text { as } \tau \rightarrow \infty, l \rightarrow 0,
$$

which in turn yields, upon returning to the original variables, equality (1.95).

Let us consider next a perturbation of the free boundary antisymmetrical with respect to $y=0$. Its inverse Fourier transform reads

$$
\begin{aligned}
R(y, t) & =\int_{0}^{\infty} f^{l}(t) \sin (l y) d l=C_{15} \int_{0}^{\infty} F(l) g\left(l^{2} t\right)\left(1+o_{l^{2} t}(1)+o_{l}(1)\right) \sin (l y) d l \\
& =C_{15} \int_{0}^{\infty} F(l) \exp (\alpha l \sqrt{t}) \exp \left(-\frac{\gamma l^{3} t}{2}\right)\left(l^{2} t\right)^{\alpha^{2}}\left(1+o_{l^{2} t}(1)+o_{l}(1)\right) \sin (l y) d l \\
& \text { as } l^{2} t \rightarrow \infty, l \rightarrow 0 .
\end{aligned}
$$

Making use of the exponential decay of the function $g$ for fixed $l>\varepsilon$ we obtain

$$
R(y, t)=C_{15} F^{n}(0) y t^{\alpha^{2}} \int_{0}^{\varepsilon} l^{n+1+\alpha^{2}} \exp (\alpha l \sqrt{t}) \exp \left(-\frac{\gamma l^{3} t}{2}\right)\left(1+o_{\varepsilon^{2} t}(1)+o_{\varepsilon}(1)\right) d l
$$

as $\varepsilon^{2} t \rightarrow \infty, \varepsilon \rightarrow 0$. Choosing $\varepsilon=1 /(\gamma t)^{1 / 3}$, we obtain

$$
|R(y, t)|>\frac{1}{2}\left|C_{15} F^{n}(0) y\right| t^{\alpha^{2}} \int_{0}^{1 / t^{1 / 3}} l^{n+1+\alpha^{2}} \exp (\alpha l \sqrt{t}) d l
$$

with $t$ sufficiently large for the $O_{\varepsilon^{2} t}(1)$ and $O_{\varepsilon}(1)$ terms in (1.98) to be negligible. Since the integral in the right-hand side diverges as $t \rightarrow \infty$, the proof of Theorem 3 is completed.

EXAMPLE. Let the initial front displacement be

$$
R(y, T)=y \exp \left(-\frac{y^{2}}{2}\right)
$$

and the initial perturbation of the temperature satisfy the condition

$$
\phi^{l}(y, T)=0 .
$$

Graphs of $R(y, t)$, obtained from Eq. (1.98), with the constant $C_{15}$ found numerically, are presented in Fig. 1 (see p. 354) for $t=T=10, T+100, T+1000, T+5000$. 


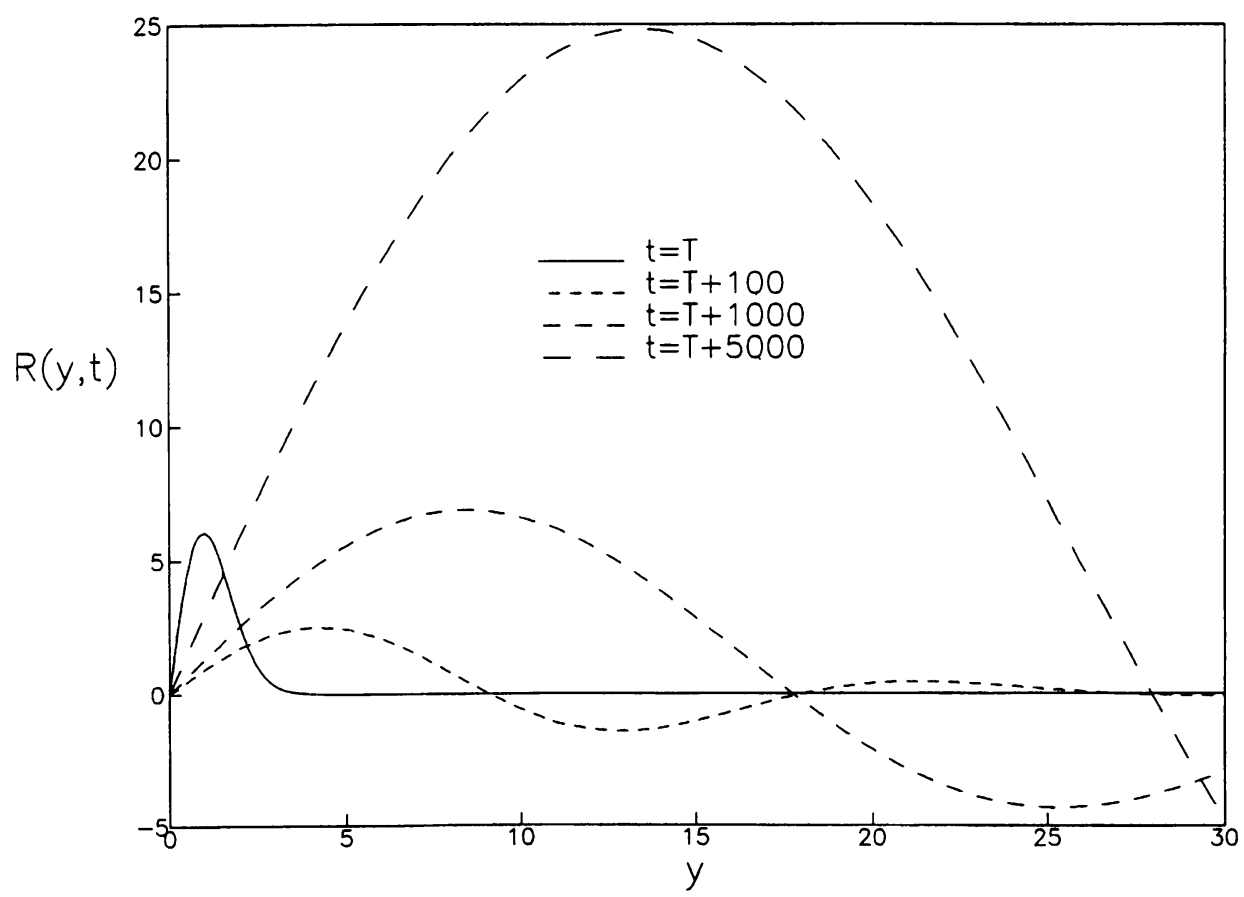

FIG. 1. Graphs of $R(y, t)$ for different time moments

Conclusions. Planar solidification is morphologically unstable also in the presence of finite surface tension. The necessary condition for stability is vanishing at the zero wave number of the Fourier transform of the initial perturbation of the free boundary together with all its derivatives. The respective sufficient condition is the absence of the long-wave modes in the initial free-boundary perturbation.

\section{REFERENCES}

[1] J. S. Langer, Instabilities and pattern formation in crystal growth, Rev. of Modern Physics 52 (1), $1-28(1980)$

[2] W. W. Mullins and R. F. Sekerka, Morphological stability of a particle growing by diffusion or heat flow, J. Appl. Phys. 34, 323-329 (1963)

[3] L. Rubinstein, Global stability of the Neumann solution of the two-phase Stefan problem, IMA J. Appl. Math. 28 (3), 287-300 (1982)

[4] J. Chadam and P. Ortoleva, The stabilizing effect of surface tension on the development of the free boundary in a planar one-dimensional Cauchy-Stefan problem, IMA J. Appl. Math. 30 (1), 57-65 (1983)

[5] W. Gautschi, Error function and Fresnel Integrals, in Handbook of Mathematical Functions, ed. by M. Abramowitz and I. A. Segun, Dover Publ., New York, 1968 